\title{
A new low-feed chip breaking tool and its effect on chip morphology
}

\author{
Manuela Pacella ${ }^{1}$
}

Received: 4 February 2019 / Accepted: 29 May 2019 / Published online: 17 June 2019

(C) The Author(s) 2019

\begin{abstract}
This paper investigates the influence of cutting conditions on the formation mechanism of chips using a tungsten carbide in-house lasered cutter (grooved chip breaker) and a benchmark commercial cutter during turning of AISI1040 medium carbon steel. Microstructure of the free surface and segment underside the chips are experimentally characterised via scanning electron microscopy (SEM) and white light interferometry. The mechanism of chip formation is classified into continuous, partially segmented, segmented and discontinuous. Chip breaking ability is achieved for all tested feed speeds at depth of cut above $1.2 \mathrm{~mm}$, marking the transition from continuous to segmented chips. The chip breaker manufactured via a nanosecond laser proves to enable for the first time breaking of the chip below a feed rate of $0.1 \mathrm{~mm} / \mathrm{rev}$ outperforming the commercial cutter and showing viability for the production capabilities of lasers for mass manufacture. Lamellae-type chips are revealed from machining using the lasered tool, while brush-stroke chips are discovered and introduced for the first time from machining using the benchmark cutter. While the lamellae form from cleavage cracks due to strain incompatibility at inclusions caused by an excess in critical shear strain. The brush-stroke chips are caused by a localised increase of temperature at the tool/material interface which lead to thermal softening of the workpiece: the resulting surface experiences large areas of plastic deformation. For the in-house lasered tool, at higher cutting speed, the shear strain hardening reduces the flow stress of the workpiece material in the shear zone.
\end{abstract}

Keywords Chip morphology $\cdot$ Turning tools $\cdot$ Chip breaker $\cdot$ Laser manufacturing $\cdot$ Shear strain hardening $\cdot$ Thermal softening

\section{Introduction}

Controlled chip flow is a requirement for automated manufacturing systems to improve productivity [1]. Thus, chip breakers have become essential features for chip management. This is achieved by manipulating the chip flow through chip breaker design (groove or obstacles on the rake face) and selecting appropriate cutting conditions. Desired examples of chip management include ensuring that chips do not interfere with the cutting operation by preventing chips from colliding with the workpiece or tool, hindering the surface finish or causing premature tool wear. Good chip management also ensures safety in machining operations. Groove-type chip breakers operate by inducing a bending stress on a chip by decreasing the radius of curvature of the chip, causing the chip

Manuela Pacella

M.Pacella@lboro.ac.uk

1 Wolfson School of Mechanical, Electrical and Manufacturing Engineering, Loughborough University,

Loughborough, Leicestershire LE11 3TU, UK to curl [2]. The chip breaks when the stress at the root of the chip overcomes the fracture strength of the chip [2]. Groovetype chip do not require setup, increase the effective rake angle, and reduce tool-chip contact length which promotes reduced cutting forces and increased tool life when compared to plain cutters. Obstacle-type chip breakers have an infinite number of configurations and operate by the same principle of groove-type chip breakers: fracture occurs by decreasing the radius of the curvature of the chip. Jawahir and Fang [3] developed a knowledge-based system for designing chip breakers and analysed the effects of machine conditions, tool geometries and chip breaking geometry in oblique machining and concluded that effective chip breaking at minimum power consumption can be achieved through the optimum groove utilisation of the chip breakers. Sreekala [4] compared two types of chip breaker geometry, vee-groove and curved groove with six designs investigated: a primary finding was that curved grooves proved better in chip control at low feeds due to forced curling and a higher chip tool contact length causing the chip to curl tighter and break with the curvature of the groove. This finding should be used for designing chip breakers at feed rates lower than $0.1 \mathrm{~mm} / \mathrm{rev}$. Gurbuz et al. [5] 
compared chip breaker geometry and its effect on cutting forces for oblique cutting on a medium carbon steel (AISI 1050) workpiece. It was found that the tool with highest geometric complexity experienced the highest cutting force, emphasising that simpler design might be preferable for extending tool life time. The same finding was confirmed by Ali and Murugan [6], who investigated the influence of chip breaker groove width and angle in turning low carbon steel. Using the length of the chip as effectiveness parameter, they found that chip breakers with the most complex geometry caused the highest frictional forces. Lotfi et al. [1] studied the effects of chip breaker geometry on cutting force, chip shape, and bending moment using finite element and experimental methods on AISI 1045 steel. Analysis of bending moment revealed that the depth of the chip breaker and the length of land have a major contribution on obtaining short chips. They found that groove-type chip breakers are preferable for lowering cutting forces and producing favourable chip shapes. Moreover, insert without chip breaker and with more complex chip breaker geometry generate the longest and shortest chips, respectively, where both could be hazardous to the operator. Analysis of bending moment revealed that inclination of surface, length of land and depth of chip breaker geometry had the highest effects on obtaining short chips.

Sreekala and Visweswararao [4] discussed the methodology of designing chip breaker geometry at low feed rates with the aim of inducing strain in the chip greater than its fracture strain. Finite element modelling was used to investigate the effectiveness of $\mathrm{V}$-shaped and curved grooves on fracture strain. It was found that curved grooves were better. Qibiao et al. [7] characterised the chips of AISI 1045 steel under different cutting speeds $(100-3600 \mathrm{~m} / \mathrm{min})$. They identified two types of chips: continuous and serrated. They revealed that in the continuous chip formation mechanism, the shear strain and shear strain rate both increase with the cutting speed and that the influence of the cutting speed on the shear strain rate is more sensitive than that on the shear strain. They also discovered that in the serrated chip formation mechanism the frequency of serration, the degree of segmentation, the shear strain rate and the shear strain all increase with the cutting speed. Gonzalo et al. [8] experimentally evaluated the chip shape for different cutting conditions and tool angles. They also conducted two-dimensional and 3D finite elements simulations to obtain the optimal design of the chip breaker geometry, which was engraved into the insert by laser micromachining. This reduced the chip size, obtaining improved surface roughness and removing the chip material from the cutting zone. Laser machining has emerged to be an attractive method for producing chip breakers. Localised thermal energy is targeted in a focused beam area on the workpiece and the required shape is achieved via a thermo-mechanical removal process. This technique enables the manufacturing of micro geometries in tight tolerances on most difficult to cut materials $[9,10]$ with minimal thermal damage $[11,12]$. Process by laser shows advantageous surface quality benefits over grinding and electro discharge machining (EDM) as it is a non-contact process with minimal heat affected zones when using short $(<100 \mathrm{~ns})$ pulse durations [13].

Many researches have been conducted on chip breaker design in tungsten carbide inserts and some of these involved laser technologies as the manufacturing process. To the best of the authors' knowledge there is a gap in the analysis of the chip morphology when laser technology is employed for the manufacturing of the chip breaker. Furthermore, the efficiency of laser-manufactured chip breakers in low feed speed applications with the aim of inducing strain in the chip greater than its fracture strain has never been investigated. Therefore, the development of a lasered chip breaker to investigate its performance and benchmark for use in cutting tool applications becomes essential, particularly considering that turning operations using $\mathrm{WC}$ are an integral part of precision machining operation for automotive and aerospace components.

The aims of this work are twofold. Firstly, the design of a chip breaker based on Fang's original chip breaker geometry [14] is proposed and a new laser-based process introduced for its manufacture to enable for the first time breaking of the chip in turning below feed rates of $0.1 \mathrm{~mm} / \mathrm{rev}$. Secondly, the mechanism of chip formation and chip breaking-ability is investigated as a function of feed speed ( $\mathrm{f}[\mathrm{mm} / \mathrm{rev}]$ ), depth of cut (d.o.c. $[\mathrm{mm}])$ and cutting speed $(\mathrm{Vc}[\mathrm{m} / \mathrm{s}])$ for the proposed design and a commercial benchmark leading to the discovery of a different chip formation mechanism for the laser manufacture tools.

\section{Methodology}

\subsection{Chip breaker design}

The design of chip breaker selected in this study is based on Jawahir and Fang's design criteria [5]. A curved symmetrical groove profile is designed to allow (1) machining at midconditions $(f=0.18 \mathrm{~mm} / \mathrm{rev}$; d.o.c $=1.6 \mathrm{~mm})$ and $(2)$ achieving chip breaking below $f=0.15 \mathrm{~mm} / \mathrm{rev}$ limit stated by previous research $[14,15]$. Since chip breaking effectiveness is better at higher feed and depths of cut, the design and performance of the lasered chip breaker are tested at extreme conditions; thus, the design targets lower feed rates and depths of cut for finishing operations.

The following design steps are considered: (1) selection of groove size and profile, (2) identification of tool restricted contact length land width $(h)$ and predicted natural tool chip contact length $\left(h_{n}\right)$. ), (3) identification of predicted chip 
sideflow angle $\eta_{s}$, (4) optimisation of land width, (5) selection of final dimensions (predicted chip backflow angle $\eta_{b}$, groove radius and back wall height).

Based on previous research [14], for feed rate $0.15<\mathrm{f}<$ $0.3 \mathrm{~mm} / \mathrm{rev}$ (design feed rate of $0.18 \mathrm{~mm} / \mathrm{rev}$ ), a groove width $(B)$ of $1.5 \mathrm{~mm}$ is selected, with a land width given in Eq. (1)

$h=0.6 h_{n}$

A raised backwall feature is selected as chip profile. Fatima et al. [16] source multiple equations to model the natural contact length and propose that the most accurate equation is reported by Poletika [17] and it is expressed in Eq. (2)

$h_{n}=t_{1}(2.05 \xi-0.55)$

where $t_{1}$ is the uncut chip thickness, and for oblique cutting ( $75^{\circ}$ toolholder), this is equivalent to Eq. (3) from previous research [8].

$t_{1}=f \sin 75$

and $\xi$ is the chip thickness ratio [16] which is given by Eq. (4)

$\xi=\frac{\text { chip thickness }}{\text { uncut chip thickness }}$

The chip thickness value is assumed to be $0.347 \mathrm{~mm}$, achieved from experiments on a AISI 1045 workpiece $(f=0.2 \mathrm{~mm} / \mathrm{rev}$; d.o.c. $=2.12 \mathrm{~mm})$ in a research conducted by Jawahir [8]. The uncut chip thickness $t_{1}$ is calculated using Eq. (5), and the chip compression ratio $\xi$ using Eq. (6)

$t_{1}=0.2 \sin 75=0.18 \mathrm{~mm}$

$\xi=\frac{0.347}{0.18}=1.93$

The natural contact length $h_{n}$ is found to be $0.613 \mathrm{~mm}$ using Eq. 2 and contact length $h$ is $0.368 \mathrm{~mm}$ by Eq. (1). The chip sideflow angle $\eta_{s}$ for oblique cutting is derived from Jiang et al. [18] and given in Eq. (7)

$\eta_{s}=0.208$ (d.o.c. $)^{-0.744} f^{0.424}(r+0.45)^{0.682}(x-16)^{1.28} 0.988^{\gamma}+0.62 \lambda$

where $r$ is the tool nose radius $(0.8 \mathrm{~mm}), x$ is the principle cutting edge angle $\left(75^{\circ}\right), \gamma$ is the insert rake angle $\left(0^{\circ}\right)$ and $\lambda$ represents the inclination angle $\left(15^{\circ}\right)$. A feed rate of $0.18 \mathrm{~mm} /$ rev and a depth of cut of $0.4 \mathrm{~mm}$ are selected for the design, leading to a chip sideflow angle of $52.1^{\circ}$.

The cosine of the sideflow angle is applied to the restricted tool contact length. This reduces the physical restricted tool contact length, in operation with the sideflow angle; however, the restricted tool contact length is increased as the sideflow goes to $52^{\circ}$. This is to ensure the restricted tool contact length remains smaller than the natural contact length. A new value of contact length $h$ is calculated to be $0.226 \mathrm{~mm}$ from Eq. (8) based on the sideflow angle $\eta_{s}$

$h_{1}=h \cos \eta_{s}$

The smaller contact length has better chip breakability due to a smaller upcurl radius [14], where the groove is being used more effectively with a smaller land width. For this reason, a land width of $0.18 \mathrm{~mm}$ is selected for its better chip breakability. and this is below the previous calculated values of contact length $h=0.226 \mathrm{~mm}$.

Interpolation from data in existing literature is used to determine the chip backflow angle $\eta_{b}$. Literature data include two graphs plotting $t_{1} / h$ against $\eta_{b}$ both for orthogonal cutting [5] and oblique cutting [15] at a $40^{\circ}$ cutting angle. For the orthogonal graph, representation is made in respect of friction parameter $\tau / k$ where $\tau$ is the shear stress and $k$ is the flow stress. $\tau / k$ is interpolated for the previously calculated design value of $t_{1}$ and the value of $\eta_{b}(1)$ is interpolated between fixed graph lines of $\tau / k=0.75$ and $\tau / k=1.0$, and 0.5 and 0.75 in the case of $\eta_{b}(2)$ at a fixed $\tau / k=0.65$. Jawahir found that there was a drop in $\tau / k$ to 0.65 at $h=0.2 \mathrm{~mm}$. Therefore, $\eta_{b}(1)$ is calculated for reducing $\tau / k$ with feed rate and $\eta_{b}(2)$ calculated for a fixed $\tau / k$ of 0.65 since the design is to use a restricted contact length of $h=0.18 \mathrm{~mm}$ whose value lays in the vicinity of $h=0.2 \mathrm{~mm}(\tau / k=0.65)$ as shown in Table 1 . An evaluation of $\tau / k$ variation with $t_{1}$ for this decrease at $h=0.2 \mathrm{~mm}$ is not available. For the designed feed rate of $0.18 \mathrm{~mm} / \mathrm{rev}$, the toolholder gives an oblique cutting angle of $15^{\circ}$. Interpolating for the actual and graphical oblique angles of $15^{\circ}$ and $40^{\circ}$ against $15^{\circ}$ and $18.5^{\circ}$ provides a value of backflow angle of $16.3^{\circ}$.

For grooved profiles, the groove tangent angle $\theta$ related to the chip backflow angle can be defined as follow.

If $\left(\eta_{b}-\alpha\right)<\theta$, the chip cannot fully use the groove

If $\left(\eta_{b}-\alpha\right)>\theta$, the chip will overuse the groove profile,

where $\alpha$ is the insert rake angle.

For the design proposed in this study, the best use of the groove is exploited; thus, the use of the ratio in Eq. (11) firstly introduced by Fang [15] is utilised.

$\left(\eta_{b}-\alpha\right) / \theta=1.2$

Therefore, groove entry angle $\theta$ is calculated to be $13.6^{\circ}$.

The groove radius $R_{o}$ is calculated to be $3.19 \mathrm{~mm}$ based on Eq. (12) from previous work [14].

$R_{o}=\frac{B}{2 \sin \theta}$ 
Table 1 Values for the prediction of $\eta_{b}$

\begin{tabular}{lllllllll}
\hline$f(\mathrm{~mm} / \mathrm{rev})$ & $t_{1}(\mathrm{f} \sin 75)$ & $\mathrm{h}$ & $t_{1} / h$ & $\tau / k$ & $\eta_{b}(1)^{\circ}$ & $\eta_{b}(2)^{\circ}(\tau / k=0.65)$ & $\eta_{b, \text { exp } .}{ }^{\circ}$ & $\eta_{b, \text { the. }}{ }^{\circ}$ \\
\hline 0.1 & 0.096 & 0.18 & 0.54 & 0.97 & 11 & 3 & 9 & 12 \\
0.18 & 0.174 & 0.18 & 0.97 & 0.87 & 20 & 15 & 18.5 & 22 \\
0.2 & 0.193 & 0.18 & 1.07 & 0.84 & 20 & 17 & 19 & 22.5 \\
0.25 & 0.241 & 0.18 & 1.34 & 0.825 & 28 & 25 & 25 & 29 \\
0.35 & 0.338 & 0.18 & 1.88 & 0.81 & 42 & 42 & $>37.5$ & $>27.5$ \\
\hline
\end{tabular}

Groove depth $d$ is calculated to be $0.09 \mathrm{~mm}$ based on [14] in Eq. (13)

$d=R_{o}-\sqrt{R_{o}{ }^{2}-\left(\frac{B}{2}\right)^{2}}$

For the design, a groove depth of $0.10 \mathrm{~mm}>0.09 \mathrm{~mm}$ is selected.

Based on the design proposed by Fang [14], the backwall height $h_{b}$ should follow Eq. (14)

$h_{b}=0.5 d$

However, there is no qualification for Fang's use of this 'rule' to determine back wall height, it can only be assumed that this was a measured criteria from the selection of cutters. Fang based design rules on. It is to be noted that the selection of cutters was specified for higher ranges of feed rate and d.o.c. In order to meet criteria of lowering the critical feed rate and achieve a feed rate of $0.15 \mathrm{~mm} / \mathrm{rev}$, consideration is given to increasing the back-wall height. At this point, the design is modified from Fang research. Back wall height is selected as $0.1 \mathrm{~mm}(100 \mu \mathrm{m})$ which is four times greater than Fang criteria.

The final parameters for the designed chip breaker to be laser manufactured are reported in Table 2.

\subsection{Chip breaker manufacture}

A $20 \mathrm{~W}$ single mode SPI pulsed Yb-doped fibre laser (1064 nm wavelength) based on a MOPA amplifier was used to manufacture the chip breaker on the tungsten carbide plain cutter. Programmed waveforms which result from an optimization of the peak power at a specific pulse repetition rate based on direct modulation of the seed laser are used. The waveforms are the results of an optimization of the peak power at a specific pulse repetition rate. Waveform 28 (repetition rate at which the peak power is optimised, $290 \mathrm{kHz}$; pulse duration 15, ns; maximum pulse energy, 0. $068 \mathrm{~mJ}$ ) in Op2, Op5 and Op8. Waveform 26 (repetition rate at which the peak power is optimised, $170 \mathrm{kHz}$; pulse duration 25, ns; maximum pulse energy, 0. $12 \mathrm{~mJ}$ ) in Op10. Laser parameters and ranges for the experiments are reported in Table 3.
Figure 1 represents the Computer-Aided Design (CAD) which is input as a file.stl in the laser software to achieve the final product through integration with a Computer-Aided Manufacturing (CAM) system.

\subsection{Cutting tool materials and workpiece selection}

Selected workpiece material is a round bar (diameter $100 \mathrm{~mm}$, length $300 \mathrm{~mm}$ ) of AISI1040 medium carbon steel (EN8) whose chemical composition is reported in Table 4, supplied by Acenta Steel. EN8 steel corresponds to the BS 970-1955 standard grade and it is referenced as equivalent and similar to AISI 1045. EN8 is a medium carbon steel used in automotive industries for gears, medium torque shafts, studs, bolts, connecting rods, crankshafts and couplings [19]. It also finds wide varieties of application for forging and casting along with low cost die material in tool and die making industries [20]. The rationale behind the choice of application (turning) and workpiece material (AISI1040) resides in the scope of the paper to provide a direct comparison with previous literature in the field of chip morphology characterisation [20] and provide the readers with practical findings in applying laser technology for the manufacture of indexable ISO inserts in the wider field of turning for the precision machining of automotive components. Some example applications where this material is utilised in automotive industry are roughing of connecting rods through heavy-duty turning, or machining of journals flange and tail in the crankshaft through turning operations.

The selected tool holder is a WNT IsoClamp PSBN $75^{\circ}$ 2525 M12-T (right handed).

Selection of the cutting inserts is based on testing conditions, in-house laser manufacturing, workpiece material and to provide a reliable benchmarking test. An $\mathrm{S}$ shape is selected for all three inserts with a nose radius of $0.8 \mathrm{~mm}$. This is wider than a V shape insert to allow testing of medium depths of cut (up to $0.3 \mathrm{~mm}$ ) and guaranteeing strong edge. Furthermore, a

Table 2 Final dimensions of the chip breaker design

\begin{tabular}{llllll}
\hline $\mathrm{B}(\mathrm{mm})$ & $\mathrm{h}(\mathrm{mm})$ & $\mathrm{R}_{\mathrm{o}}(\mathrm{mm})$ & $\mathrm{d}(\mathrm{mm})$ & $\theta\left(^{\circ}\right)$ & $\mathrm{h}_{\mathrm{b}}(\mathrm{mm})$ \\
\hline 1.5 & 0.18 & 3.19 & 0.1 & 13.6 & 0.1 \\
\hline
\end{tabular}


Table 3 Process parameter range

\begin{tabular}{|c|c|c|c|c|c|c|c|c|c|c|}
\hline Process parameters & Op1 & Op2 & Op3 & Op4 & Op5 & Op6 & Op7 & Op8 & Op9 & Op 10 \\
\hline Intensity (\%) & 80 & 80 & 80 & 60 & 60 & 60 & 40 & 40 & 40 & 60 \\
\hline Repetition rate $\mathrm{f}(\mathrm{kHz})$ & 35 & 290 & 90 & 35 & 290 & 90 & 35 & 290 & 90 & 170 \\
\hline Waveform & 0 & 28 & 23 & 0 & 28 & 23 & 0 & 28 & 23 & 26 \\
\hline Pulse duration $\tau$ (ns) & 220 & 15 & 55 & 220 & 15 & 55 & 220 & 15 & 55 & 25 \\
\hline Maximum energy/pulse (mJ) & 0.57 & 0.07 & 0.22 & 0.57 & 0.07 & 0.22 & 0.57 & 0.07 & 0.22 & 0.12 \\
\hline Fluence $\psi\left(\mathrm{J} \mathrm{cm}^{-2}\right)$ & 72.8 & 8.78 & 28.3 & 54.6 & 6.59 & 21.1 & 36.4 & 4.39 & 14.2 & 11.2 \\
\hline
\end{tabular}

wider nose is suitable to avoid the introduction of heat as an additional process variable: small nose angles are weaker and have smaller cutting-edge engagement, making the tool more sensitive to the effects of heat. The chip breaker geometry is selected to suit medium turning operations, such as the typical for the selected workpiece EN8.

A plain tungsten carbide tool SNMA120408 of grade UTi20T (ISO P30 Grade) is provided by Mitsubishi with 90.5 HRA hardness (Fig. 2a). The ablation fluence threshold for $\mathrm{WC}$ is reported as $2.36 \mathrm{~J} \mathrm{~cm}^{-2}$ and thermal conductivity $38 \mathrm{~W} \mathrm{~m}^{-1} \mathrm{~K}^{-1}$ [21]. An identical square shape cutter (SNMA120408, UTi20T, ISO P30 Grade) is chosen for the laser manufacture of a chip breaker. This is a non-coated tool since coating might introduce additional effects due to adhesion and differing compositions between the coating and the substrate of the tool during machining. The choice of a square geometry resides in ensuring consistency of the coordinate positioning system during operation. A commercial cutter SNMG120408EN HCN2125-NF23 with chip breaker is selected as benchmark and it is provided by WNT Ltd. The chip breaker is a groove-type which features obstacle patterns as shown in Fig. 2c. To replicate industrial scenarios, the cutting process is oblique, with $15^{\circ}$ orientation to the workpiece. Most previous chip breaker investigations have been done with orthogonal cutting edges; however, an oblique cutting edge is more relevant to industry as oblique tools are far more common, further justifying the square cutter.

\subsection{Lasered cutter testing and benchmark}

Machining trials are conducted on a XYZ Proturn SLX 1630 turning center. To ensure benchmarking can provide accurate results both in terms of chip characterisation, and in relation to the chip breaker comparison, the workpiece material, tool dimensions and cutting conditions are constant for the three cutters. The length of cut is set at $10 \mathrm{~mm}$ which is found to give a sufficient volume of chip for the characterisation. Cutting speed are varied between 1.6 and $2.6 \mathrm{~m} / \mathrm{s}$ for both lasered and WNT cutters. Feed rate is varied between 0.1 and $0.26 \mathrm{~mm} / \mathrm{rev}$, while depth of cut is varied in the range 0.4 to $2.6 \mathrm{~mm}$. This is to ensure that chip analysis and characterisation can occur in critical machining conditions and the chip breaker design can be tested in its performance conditions. A negative rake angle of $-15^{\circ}$ and a relief angle of $15^{\circ}$ are employed for all turning tests. A total of 25 test conditions are used to enable the quantitative analysis of the chip as well as chip charts both for the lasered and benchmark WNT cutters. The starting conditions are intentionally set short of the
Fig. 1 Solidwork design of the chip breaker

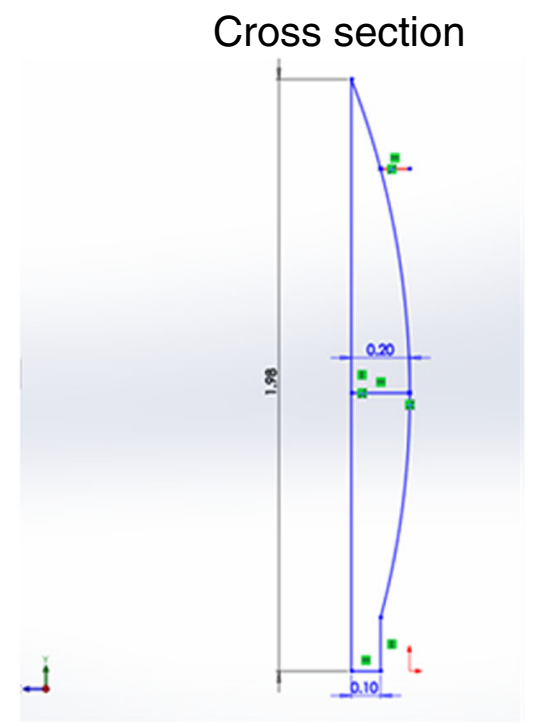

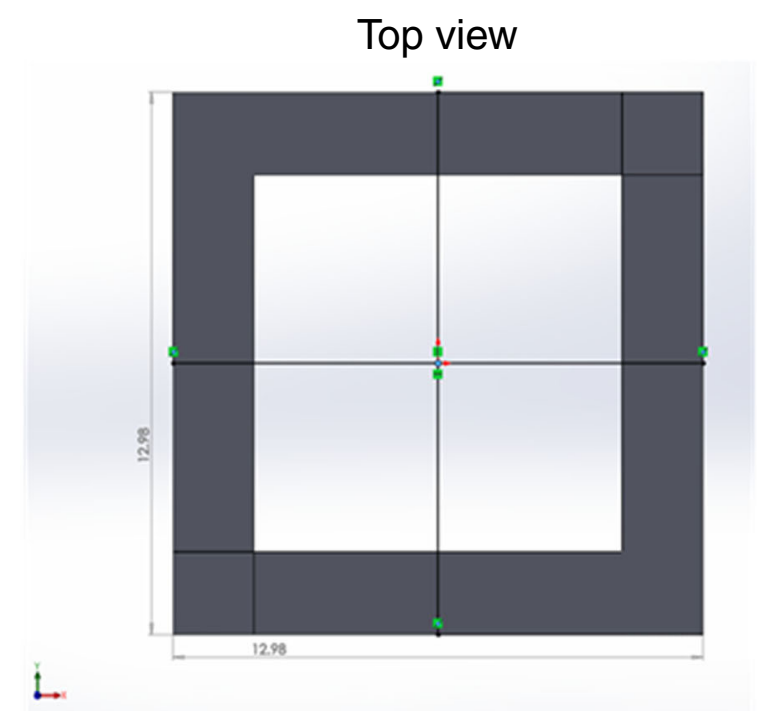


Table 4 Chemical composition of AISI 1040

\begin{tabular}{llllll}
\hline Element & $\mathrm{C}(\%)$ & $\mathrm{Mn}(\%)$ & $\mathrm{Fe}(\%)$ & $\mathrm{S}(\%)$ & $\mathrm{P}(\%)$ \\
\hline Wt.\% & $0.37-0.44$ & $0.60-0.90$ & $98.6-99$ & $\leq 0.050$ & $\leq 0.040$ \\
\hline
\end{tabular}

recommended operating range to observe the transition from continuous (long snarled) chips to segmented (half arc) chips. The plain cutter testing does not include 25 testing conditions used for the lasered and WNT, as it is expected the chip morphology will have little variation, therefore extensive testing of a plain cutter is of less significance for this study. However, some testing is conducted primarily for comparing the effect of a plain versus chip breaker profile of the same insert. Repeat tests are done to check the consistency of the results. Adobe Lightroom 4 is used to enhance the images for a clear white background. GNU Image Manipulation Program (GIMP) is used to compile the images together onto the chip chart.

\section{Results and discussion}

\subsection{Manufacture and testing}

Figure 2 shows top and flank view of all tested cutters, Fig. $2 b$ depicts the grooved chip breaker achieved through the proposed laser process. Images of the flank view of the cutters underline the geometrical differences particularly between the UTi20T in-house lasered and the WNT benchmark. Microscopic analyses of the as-received inserts revealed the cutting edges were previously treated via a combination of grinding and honing processes, resulting in chamfered edges with a chamfer width of $120 \mu \mathrm{m}$ circa. In order to produce a raised backwall starting with a plain insert (Fig. 2a), it is necessary to machine down and across the cutting edge. This is the first operation performed to machine the back wall. Previous laser cuts performed for groove optimization did not create a heat-affected zone (HAZ); however, the ablation required to create a raised surface for the backwall proved to create a debris of recast layer across the cutting edge as shown in the red circles at the corner of the cutter (Fig. 2b). This should be considered for mass manufacture and cutting tool tolerance specifications, although it would not affect the most common post-processing operations on the turning tool such as honing. Furthermore, performance testing shows that the cutting edge performs regardless.

Geometrical differences in the chip breaker profiles between the UTi20T in-house lasered and the WNT benchmark are evaluated through Talysurf surface roughness measurement and results are shown in Fig. 3. An important observation is about the width of the groove which is $1.8 \mathrm{~mm}$ for the lasered cutter and $1.4 \mathrm{~mm}$ for the WNT benchmark one. It is expected that a difference of $0.4 \mathrm{~mm}$ in the groove width will impact the chip breaking capability and cutters performance. Previous experiments [22] demonstrated that inserts with a larger land angle, higher back wall and smaller groove is more effective in chip breaking. Particularly, the width of groove is reported to give the greatest contribution to chip breaking than any other parameters [22]. At conditions of lower feed rate, it is expected that a smaller groove width will perform more efficiently. Work needs to be carried out to produce an improved surface finish within the groove (Fig. 3b).

\subsection{Effect of cutting conditions on the transition from continuous to serrated chip}

Chips collected from different test conditions are classified into continuous, partially segmented and segmented. For lasered and benchmark inserts, the continuous and segmented chips are defined on the chip chart, respectively, in Fig. 4a and $\mathrm{b}$, where these are labelled utilising nomenclature from previous research [8]. The level of acceptability of different chips (marked as green or red in Fig. 4) is based on industrial data provided in the Tungaloy Catalogue TE0707-E2 where chips are classified into shape A, B, C, D and E. For lasered insert at
Fig. 2 Optical images of UTi20T plain (a), UTi20T in-house lasered (b) and WNT with chip breaker (c)

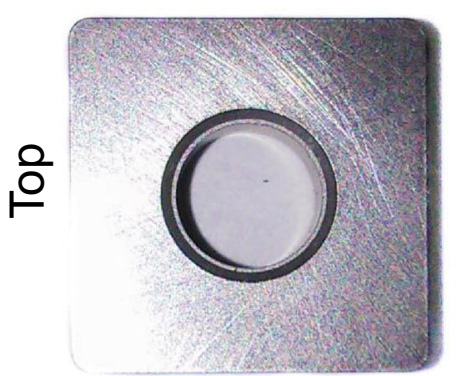

(a)

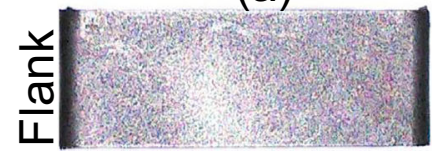

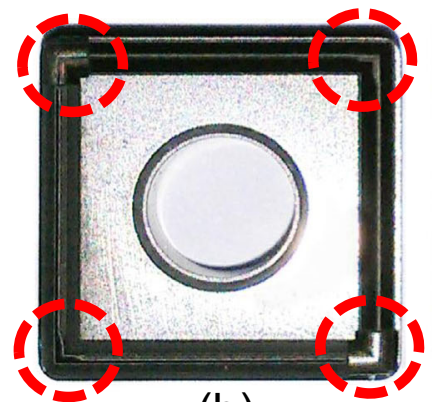

(b)

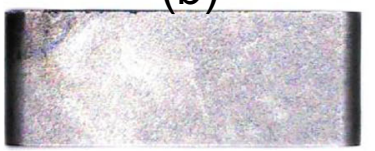

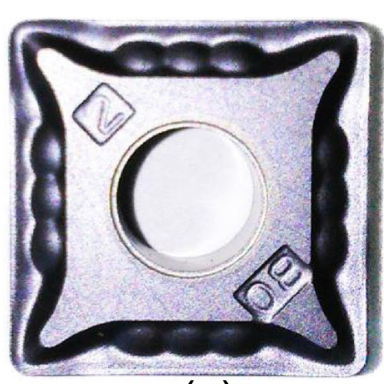

(c)

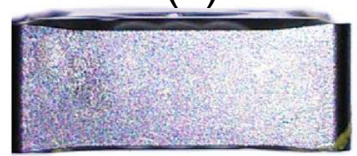


(a)

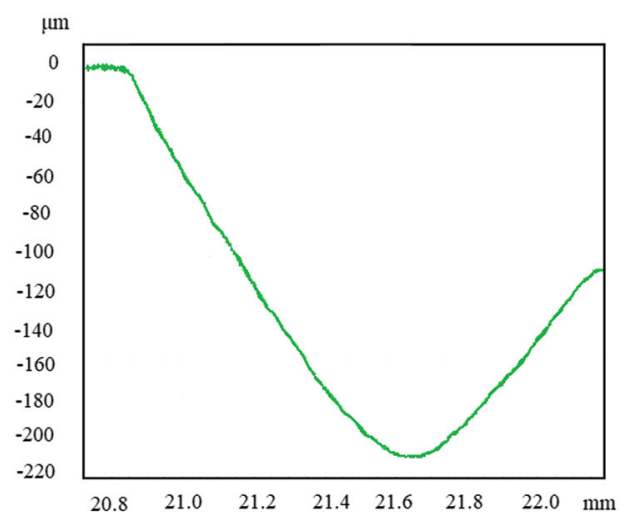

(b)

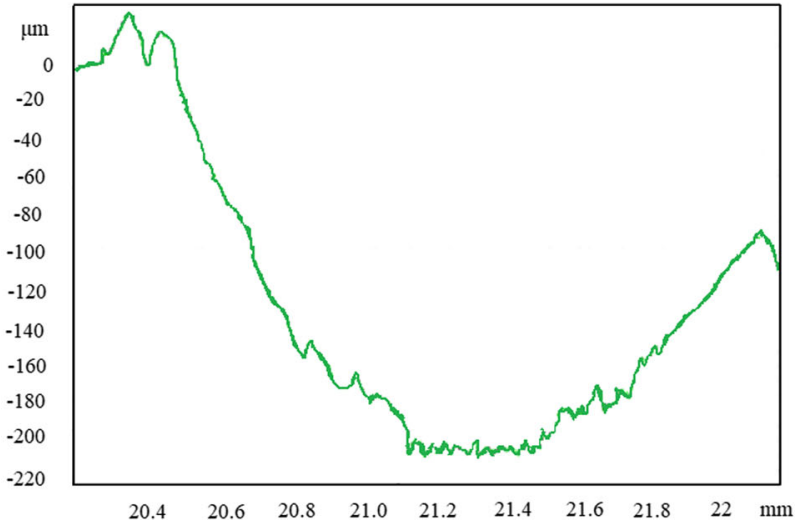

Fig. 3 Surface roughness Talysurf profile of commercial WNT chip breaker (a) and in-house laser milled (b)

Fig. 4 Chip chart achieved testing the UTi20T in-house lasered cutter (a) and the WNT benchmark cutter (b) (a)

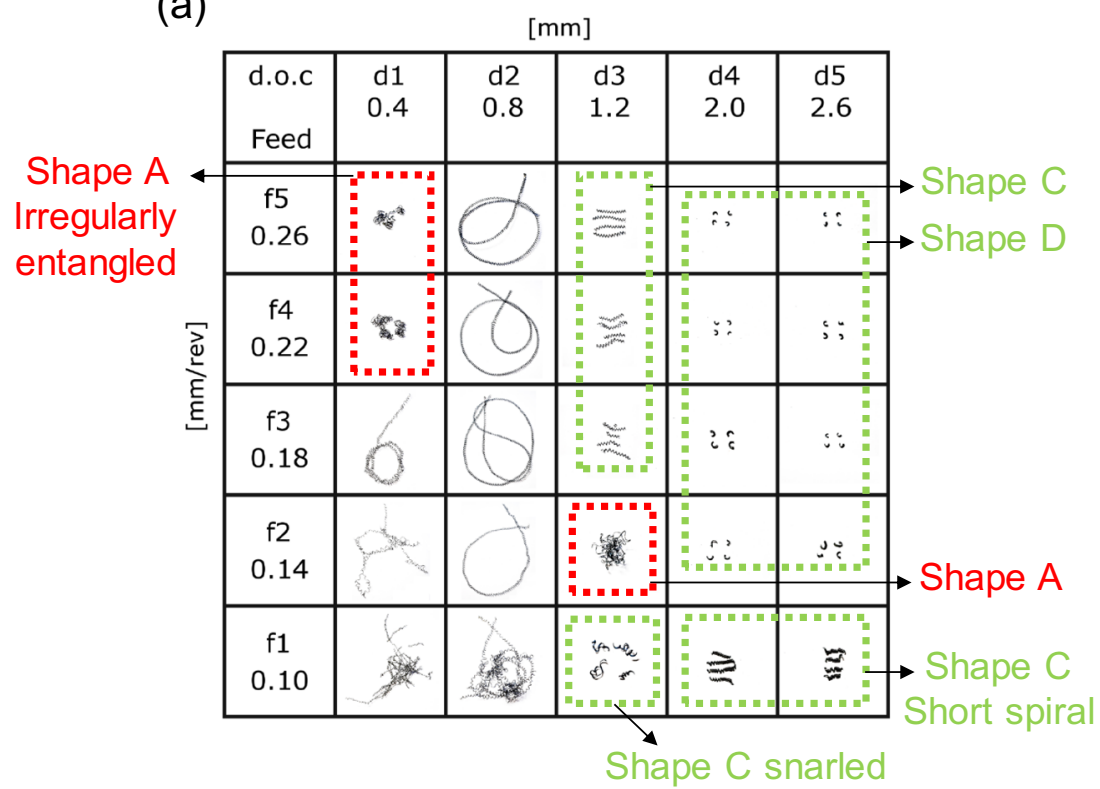

(b)

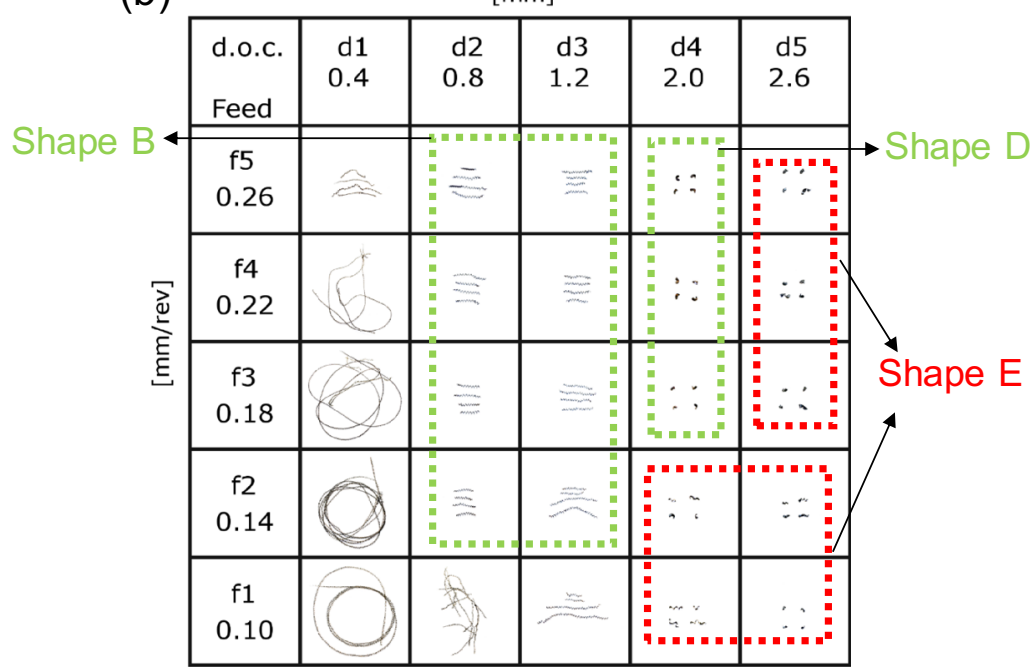


Fig. 5 Chip morphology as a function of feed speed and flank land width for two different cutters at $\mathrm{Vc}=2.67 \mathrm{~m} / \mathrm{s}(\mathbf{a})$ and at two different cutting speeds at d.o.c. $=2 \mathrm{~mm}$ using the lasered tool (b) (a)

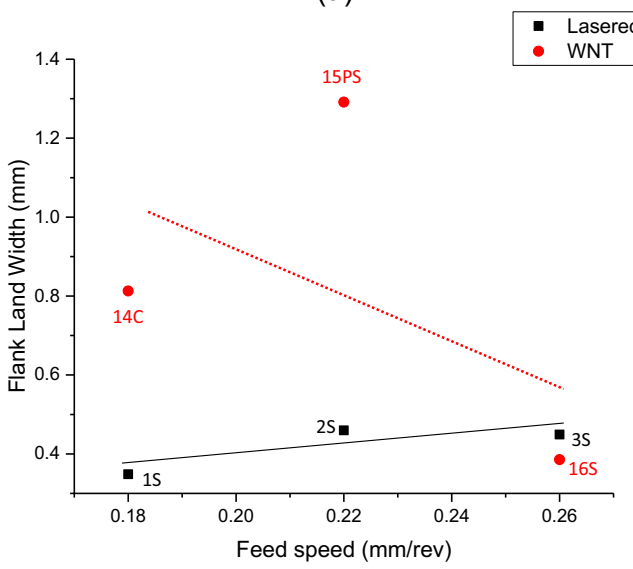

(b)

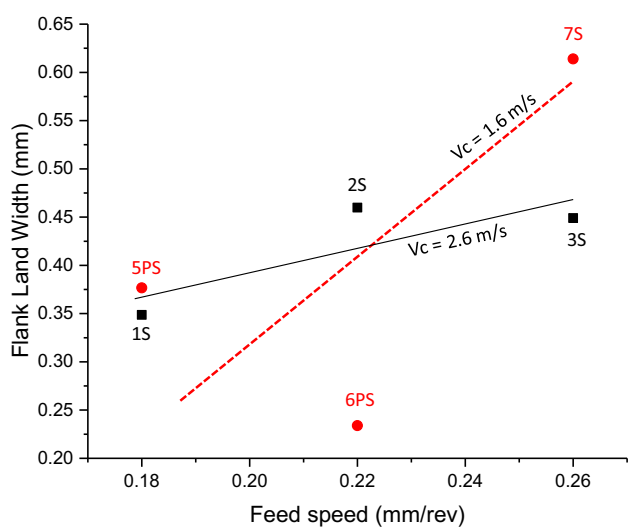

depths of cut below $1.2 \mathrm{~mm}$, the chip is continuous; above $1.2 \mathrm{~mm}$, there is a transition from continuous to segmented. For depths of cut above $2 \mathrm{~mm}$, the achieved chip is shape D (arc chip) for feed between 0.14 and $0.26 \mathrm{~mm} / \mathrm{rev}$; however, chips transition into shape $\mathrm{C}$ (short spiral) below $0.10 \mathrm{~mm} / \mathrm{rev}$. In all cases except at a feed of $0.14 \mathrm{~mm} / \mathrm{rev}$, the achieved chip is acceptable (as shown in green in Fig. 4a). The 'birds nest' at d3f2 (d.o.c. $=1.2 \mathrm{~mm}, f=0.14 \mathrm{~mm} / \mathrm{rev}$ ) are broken chips (shape A) similar to $\mathrm{d} 3 \mathrm{fl}$ (d.o.c. $=1.2 \mathrm{~mm}, f=0.1 \mathrm{~mm} / \mathrm{rev}$ ). The latter are shape $\mathrm{C}$ also known as snarled: these became entangled in the chip container and they did not snarl at the workpiece.

For the benchmark insert acceptable chips are achieved up to $2 \mathrm{~mm}$ depth of cut and above $0.18 \mathrm{~mm} / \mathrm{rev}$, where a transition from shape B (long continuous spiral chips) into shape D (arc chips) is recorded at increasing depths of cut. A depth of cut of $1.2 \mathrm{~mm}$ is the transition point for the chip from continuous to segmented. However, at high depth of cut (above $2 \mathrm{~mm}$ ) and low feed (below $0.14 \mathrm{~mm} / \mathrm{rev}$ ), the benchmark insert does not perform as well as the in-house lasered, as confirmed by production of chip in the form of shape E: chips connect in a form of wave and are responsible to cause chatter and harm the finished surface roughness. Shape E chip is typically achieved at small depths of cut.

Figure 5a depicts the influence of feed speed and flank land width on chip morphology for lasered and benchmark cutters at a cutting speed of $2.67 \mathrm{~m} / \mathrm{s}$. A clear transition from continuous $(f=0.18 \mathrm{~mm} / \mathrm{rev})$ to partially segmented $(f=0.22 \mathrm{~mm} /$ rev) and segmented $(f=0.26 \mathrm{~mm} / \mathrm{rev})$ is revealed for the benchmark WNT insert. Chips achieved using the lasered insert are all segmented and no apparent transition from continuous to segmented is recorded (Fig. 5a). However, when cutting speed is decreased to $1.67 \mathrm{~m} / \mathrm{s}$, there is a transition from continuous to partially serrated at feed speeds below $0.22 \mathrm{~mm} /$ rev (Fig. 5b). When the cutting speed is increased to $2.67 \mathrm{~m} / \mathrm{s}$, chips appear to be always serrated. At increased cutting speed $(2.67 \mathrm{~m} / \mathrm{s})$, the transition from continuous to segmented chip is faster because the propagation of adiabatic shear within the lower region of the primary shear zone occurs in a shorter time. (a)

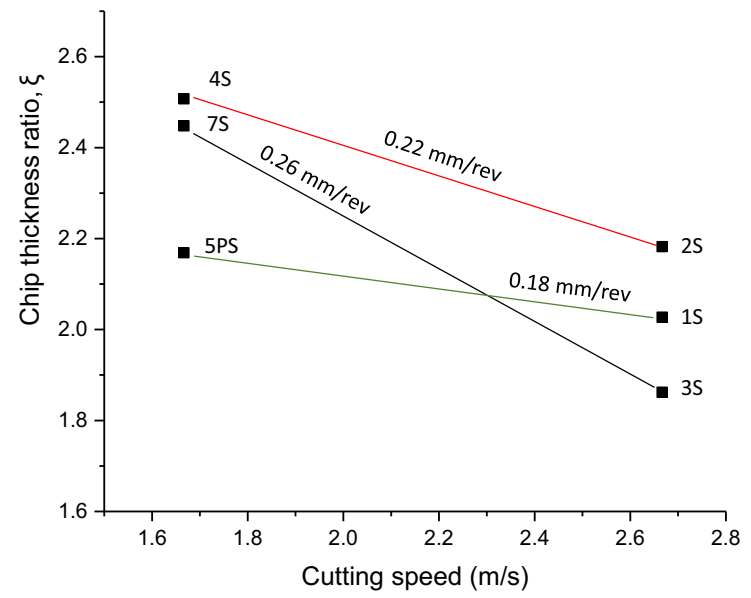

(b)

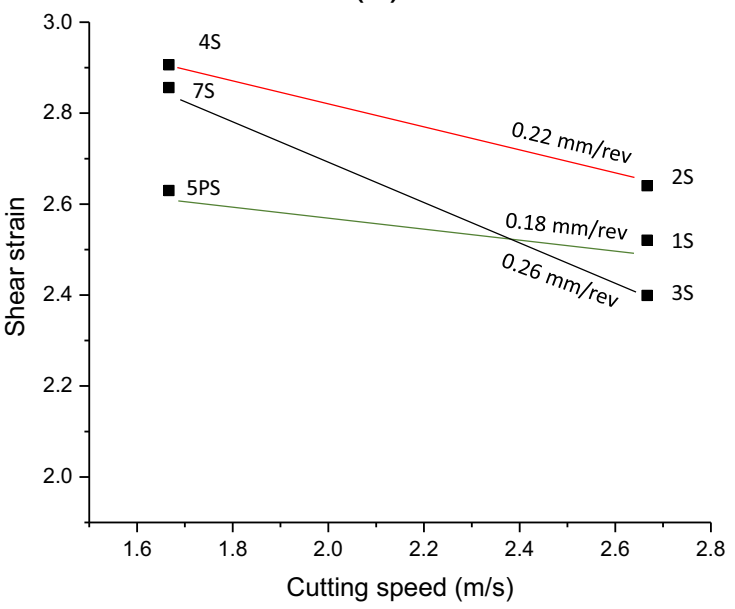

Fig. 6 Variation in chip thickness ratio (a) and shear strain (b) as a function of cutting speed for different feed speeds in the lasered tool 


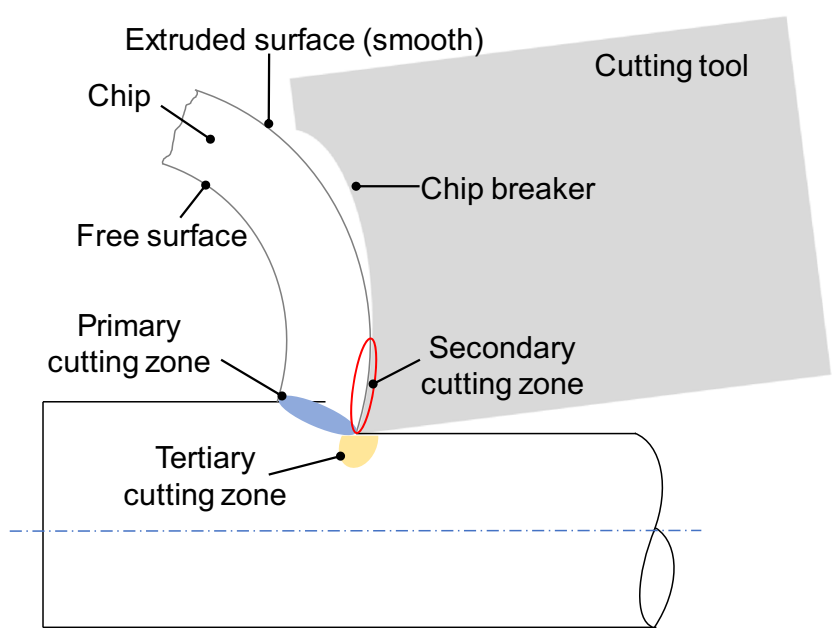

Fig. 7 Schematic of the chip formation mechanism and deformation zones

Another characteristic of the transition from continuous/ partially serrated to segmented chip is the constant decrease in the average chip thickness ratio (Fig. 6a) accompanied by a decrease in the thickness of the chips. The decrease in chip thickness ratio corresponds to an increase in shear angle and a decrease in shear strain within the primary zone as shown in Fig. 6b.

\subsection{Classification of chip and deformation mechanism}

In turning operations, three main areas of deformation can be identified: primary, secondary and tertiary deformation/ cutting zone (Fig. 11a). The primary area is at the interface between tool and workpiece where a large amount of heat is produced by shearing of the material to be removed. The heat generated in this area is directly proportional to the shear force and shearing velocity. In the secondary cutting zone, heat is generated by the friction between the tool and the chips flowing on the tool rake face. The tertiary cutting zone is defined as the area where rubbing of the tool against the machined surface occurs and it represents only $1 \%$ of the generated heat [20]. In the secondary cutting area, the force applied by the rake face of the tool on the chip exerts a similar force to a distributed load on the side of a cantilever beam; therefore, a bending moment is created [23]. The bending moment causes a difference in the velocity of the chip between the smooth surface and the free surface of the chip (Fig. 7).

For the purpose of chip characterisation, the proposed study adopts the chip classification firstly reported by Barry and Byrne [24] where two structures of free surface of chip (lamellae or folds) and two structures of segment underside (folds or dimples) are identified. Most frequently observed for both lasered and benchmark tool is the lamellar structure, however this is found on the segment underside the chips for both tools and on the free surface of the lasered one.

Figure 8 shows scanning electron micrographs of such lamellae for the in-house lasered (Fig. 8a) tool and for the benchmark one (Fig. 8b). A previous study [24] reported that for most metallic materials, the average lamella thickness is found to remain relatively constant, 2-3 $\mu \mathrm{m}$ independent of undeformed chip thickness, within the range 20-100 $\mu \mathrm{m}$.

In this study, the shear front spacing on the lamellar chip is $15 \mu \mathrm{m}$ for the lasered insert (Fig. 8a) and in the order of $20 \mu \mathrm{m}$ for the benchmark tool (Fig. 8b). The similarity between the underside of segments for the two cutters with the free surface of the lasered tool suggests that the mechanism of formation of the lamellar structure is the same. Furthermore, the directions of shear for the underside segments appear to be the same, as evidenced by the shear marks in the scanning electron micrographs in Fig. 8. The shear front spacing difference between lasered and benchmark tool can be explained considering the extent of deformation in the secondary cutting zone. The bending moment created by the rake face on the chip causes a difference in the velocity of the chip between the smooth surface and the free surface of the chip. For a cutting tool with (a)

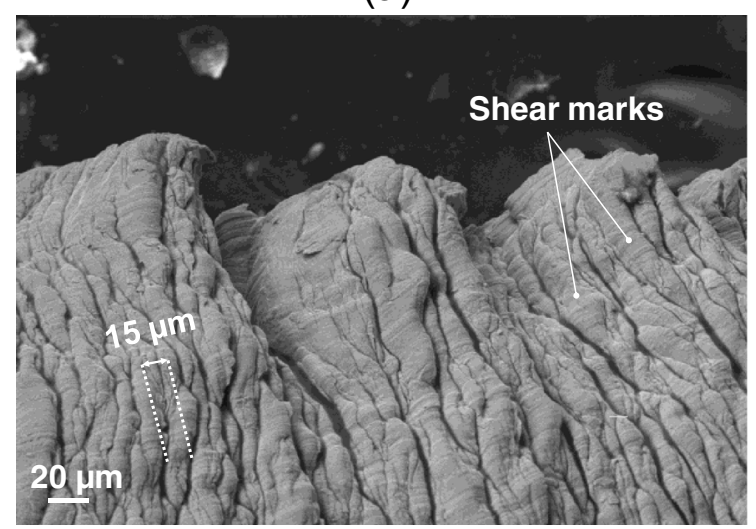

(b)

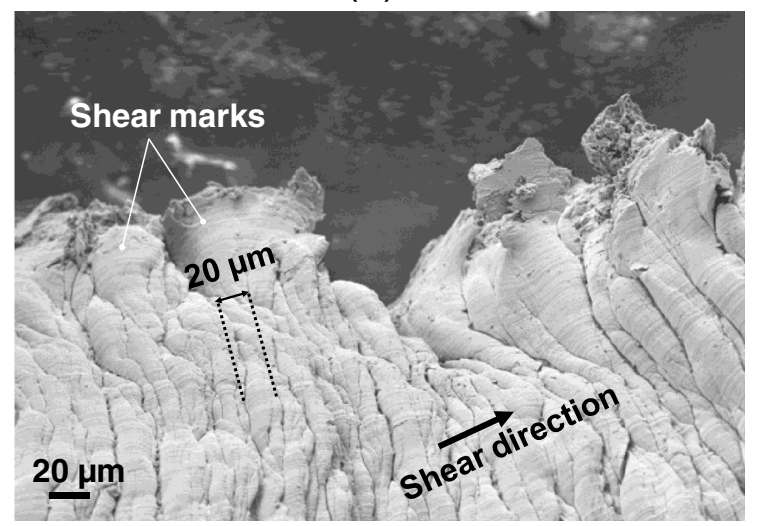

Fig. 8 Lamellae on the segment underside a serrated chip formed during cutting of AISI 1040 with $\mathrm{Vc}=2.67 \mathrm{~m} / \mathrm{s}$ and $f=0.18 \mathrm{~mm}$ using the in-house lasered tool (a) and the benchmark tool (b) 
(a)

(b)

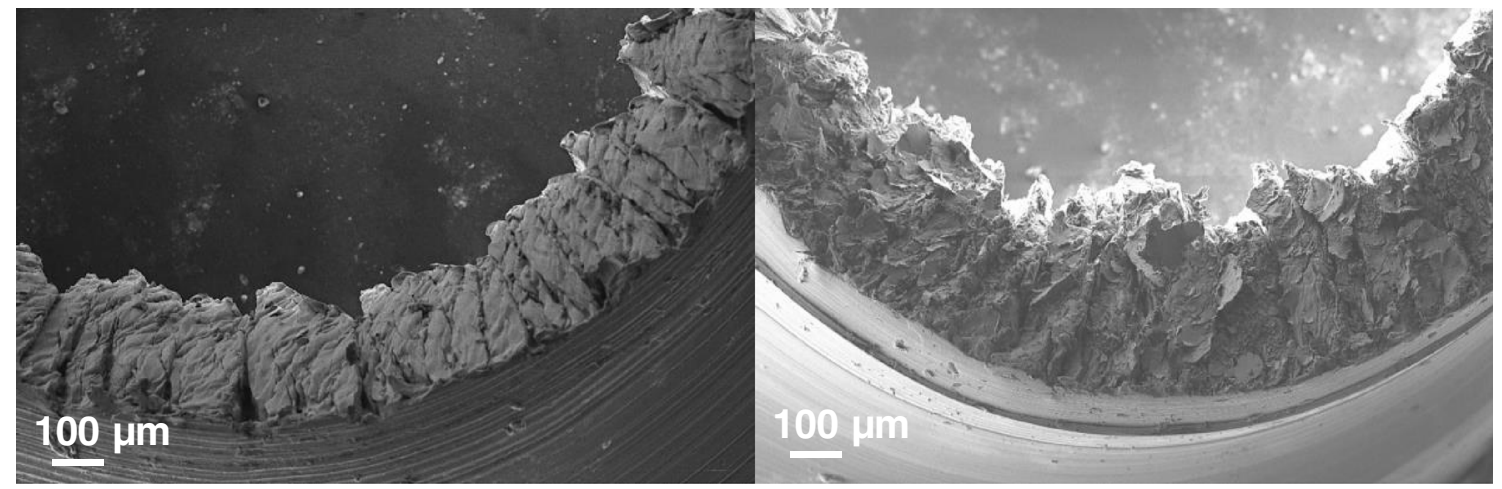

Fig. 9 Scanning electron microscopy images of a free surface of chips achieved after machining at $\mathrm{Vc}=2.67 \mathrm{~m} / \mathrm{s}$, d.o.c. $=2 \mathrm{~mm}, f=0.18 \mathrm{~mm} / \mathrm{rev}$ using lasered insert (a) and benchmark insert (b)

a chip breaker geometry the chip generated which escapes through the rake face is deformed by a compressive force exerted by the chip breaker [25]. This causes a squeezing mechanism which generates the lamellae shown in Fig. 8. Measurements of the lamellae are $15 \mu \mathrm{m}$ for the lasered insert and $20 \mu \mathrm{m}$ for the benchmark tool which show that the bending deformation is higher in the lasered tool and lower in the benchmark.

The structure of free surface of chip produced by the lasered tool is lamella-type (Fig. 9a) as expected for most metallic materials. The lamellae form from cleavage cracks due to strain incompatibility at inclusions caused by an excess in critical shear strain [24]. It is unclear from the conducted experiments weather the formation of lamellae chips both on the free surface and underside segment of chips achieved using the lasered tool is to be attributed to the laser process itself. The investigation of the subsurface damage on the cutting tool due to laser processing is out of scope for the paper; however, it is to be noted that the laser fluence utilised in this experiment is $7.5 \mathrm{~J} / \mathrm{cm}^{2}$ and that the threshold ablation fluence for Tungsten carbide is reported to be between 0.4 [26] and $2.36 \mathrm{~J} / \mathrm{cm}^{2}[21]$ depending on material specifications.

The free surface of chip produced by the benchmark cutter is not to be considered as lamella or fold, as evidenced by the scanning electron microscopy analyses. On the free surface (Fig. 9b), chip segments are not easily identified as in the lasered tool (Fig. 9a), and the deformation process appears to be of a different nature. These new structures have been termed brush-stroke. This term is derived from the similarity of the structure to paint deposited on paintings. Most likely a localised increase of temperature would lead to thermal softening on the workpiece. In this condition, cleavages or microcracks would be prevented; however, the resulting surface would see large areas of plastic deformations. This mechanism is similar to the effect of change of cutting conditions on the mode of material behaviour within the primary shear zone previously reported [24].
To confirm that all the collected chip structures are lamellar in the case of the lasered tool, polished cross sections of the chips are imaged on an optical microscope and traces of shear surfaces are found. The degree of segmentation typically increases with an increase in cutting speed. This is due to the softening of the workpiece material leading to the formation of adiabatic shear bands in the primary shear zone [7]. The experiments proposed in this paper are in contrast to previous research [7] for the in-house lasered tool. A decrease of degree of segmentation is recorded at increased cutting speed (Fig. 11a) which could be caused by a reduction of the softening mechanism on the workpiece material and consequently a reduction of adiabatic shearing in the primary zone of the chip formed. This would explain why at higher cutting speed the shear strain hardening reduces the flow stress of the workpiece material in the shear zone. This is evidenced by points 1S, 2S and 3S (Fig. 10b) which show a lower shear strain compared to $4 \mathrm{~S}$ and $7 \mathrm{~S}$ at lower cutting speed.

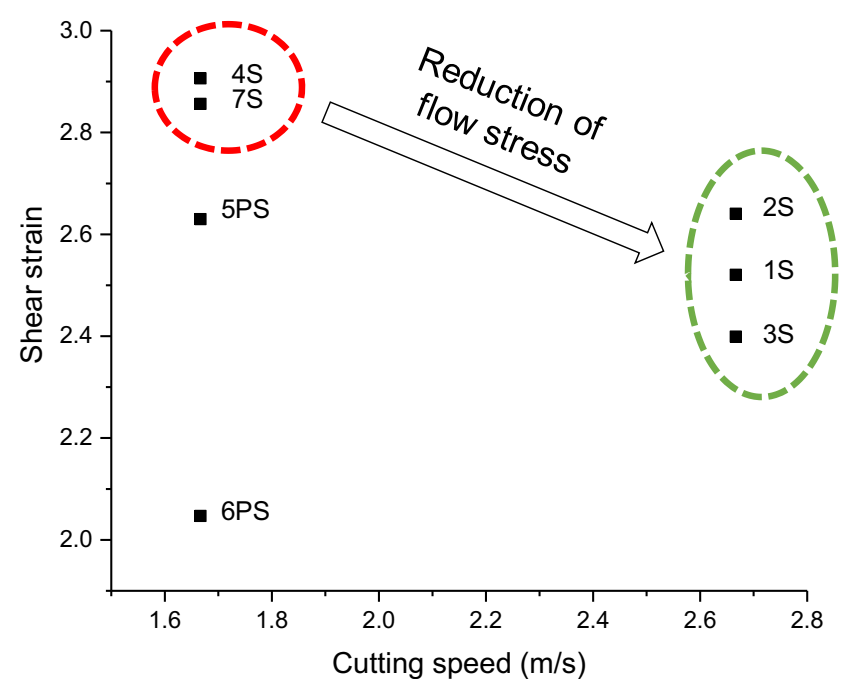

Fig. 10 Effect of cutting speed on the shear strain in the lasered tool 
Fig. 11 Degree of segmentation for the lasered cutting tool at different cutting speeds (a) and lasered versus benchmark at $\mathrm{Vc}=$ $2.67 \mathrm{~m} / \mathrm{s}(\mathbf{b})$ (a)

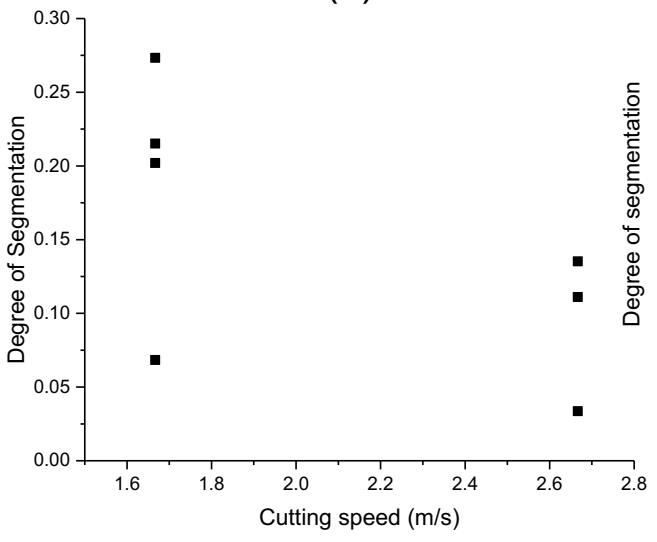

(b)

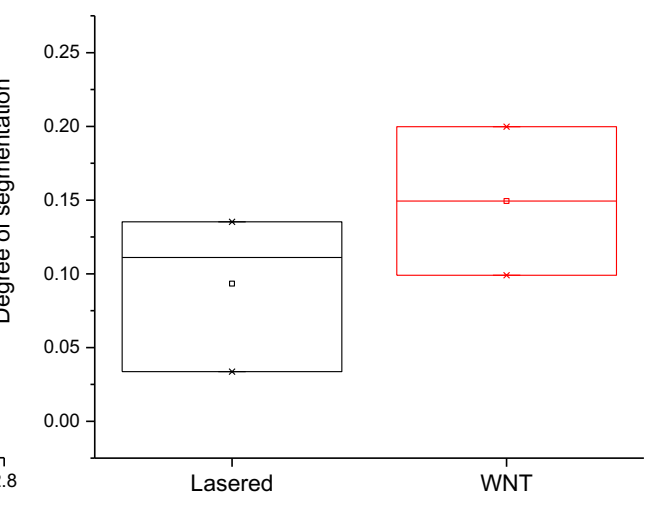

A direct comparison of the degree of segmentation in the chips achieved using the in-house lasered cutter and the benchmark (WNT) one at the same cutting speed $(\mathrm{Vc}=$ $2.67 \mathrm{~m} / \mathrm{s}$ ) shows that the degree of segmentation is lower for the lasered insert, which signify that the benchmark cutter causes the chip to experience more adiabatic shearing in the primary zone (Fig. 11b).

At lower cutting speed $(\mathrm{Vc}=1.67 \mathrm{~m} / \mathrm{s})$, the lasered tool produced an increased amount of adiabatic shear bands (regions of extreme localised deformations) in the primary deformation zone, which is located at the interface between tool and workpiece (Fig. 7). Shearing of the material to be removed creates a large amount of heat whose extent is proportional to shear force and shearing velocity. When adiabatic heating occurs, the heat generated by the deformation process is retained causing an increased local temperature. This causes thermal softening. When thermal softening prevails on the strain-hardening effect of the deformation, an instable thermo-mechanical condition of highly localised plastic shear strain may arise [27]. Crack propagation in the adiabatic shear band in the primary shear zone is depicted in Fig. 12b. The crack develops until the crack-arresting normal stress reaches a value high enough to stop crack propagation, as also suggested by model of chip formation of segmented chip proposed by Nakayama [28]. In the primary deformation area shear strain and shear strain rates are experienced as supported by the thermo-plastic shear theory [29] resulting in the formation of the segments characterising the chip (Fig. 12a). While the chip flows out of the chip breaker, due to bending deformations in the
Fig. 12 Scanning electron microscopy image of a partially segmented chip achieved through the lasered insert at $\mathrm{Vc}=$ $1.67 \mathrm{~m} / \mathrm{s}$, d.o.c. $=2.6 \mathrm{~mm}, f=$ $0.22 \mathrm{~mm} / \mathrm{rev}$ (a); void formation preceding crack nucleation and propagation in the adiabatic shear band for the same insert (b)

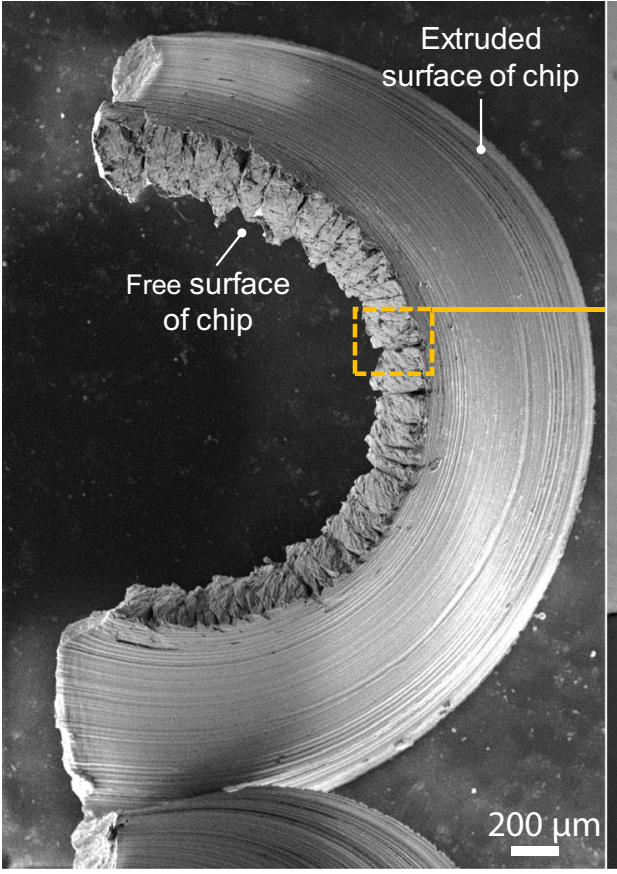

(a)

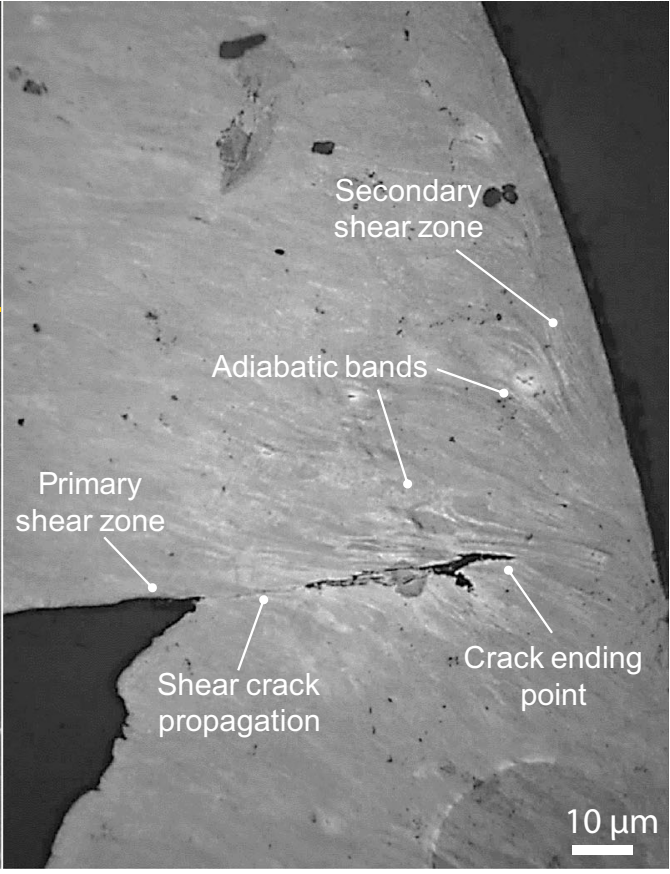

(b) 
secondary cutting zone imposed by the rake face [23] on the chip, the chip produces elastic recovery enlarging the curvature radius of the chip and increasing the strain on the free surface of the chip (Fig. 12a). When the strain becomes larger than the fracture strain of the material, the chip will fracture, as shown in Fig. 12a.

\section{Conclusions}

Both cutters show a threshold depth of cut $(1.2 \mathrm{~mm})$ for the transition of continuous into segmented chips. However, the inhouse lasered tool performs better than the benchmark one at the lowest tested feed speed $(0.1 \mathrm{~mm} / \mathrm{rev})$. It achieves acceptable short spiral chips in comparison to the ones produced by the WNT cutter which connect in a form of wave and are typically responsible to cause chatter and to harm the surface roughness. The free surface characterisation of the chip of lasered and benchmark cutters allowed to identify two different chip morphologies: lamellae-type of chips are revealed for the lasered tool and a new morphology of chip is discovered for the first time using the benchmark tool, this has been termed brushstroke type. The different morphology is caused by deformation processes of different nature: the lamellae form from cleavage cracks due to strain incompatibility at inclusions caused by an excess in critical shear strain; while the benchmark tool imposes a localised increase of temperature at the cutting tool/material interface which would lead to thermal softening of the workpiece. In this condition cleavages or micro-cracks would be prevented, however the resulting surface would see large areas of plastic deformations. For the in-house lasered tool, at higher cutting speed, the shear strain hardening reduces the flow stress of the workpiece material in the shear zone. At lower cutting speed, the lasered tool produced an increased amount of adiabatic shear bands (regions of extreme localised deformations) in the primary deformation zone. In the secondary cutting zone, the bending forces imposed by the rake face on the chip cause an enlargement of the curvature radius of the chip and an increase in the strain on the free surface of the chip.

Open Access This article is distributed under the terms of the Creative Commons Attribution 4.0 International License (http:// creativecommons.org/licenses/by/4.0/), which permits unrestricted use, distribution, and reproduction in any medium, provided you give appropriate credit to the original author(s) and the source, provide a link to the Creative Commons license, and indicate if changes were made.

\section{References}

1. Lotfi M, Akhavan Farid A, Soleimanimehr H (2015) The effect of chip breaker geometry on chip shape, bending moment, and cutting force: FE analysis and experimental study. Int J Adv Manuf Technol 78(5-8):917-925
2. Jawahir IS, Van Luttervelt CA (1993) Recent developments in chip control research and applications. CIRP Ann Manuf Technol 42(2): 659-693

3. Marimuthu S, Kamara AM, Whitehead D, Mativenga P, Li L (2010) Laser removal of TiN coatings from WC micro-tools and in-process monitoring. Opt Laser Technol 42(8):1233-1239

4. Sreekala PA, Visweswararao K (2012) A methodology for chip breaker design at low feed turning of alloy steel using finite element modelling methods. Int J Mech Eng Technol 3(2):263-273

5. Jawahir IS, Fang XD (1995) A knowledge-based approach for designing effective grooved chip breakers - 2D and 3D chip flow, chip curl and chip breaking. Int J Adv Manuf Technol 10(4):225-239

6. Ali J, Murugan M (2009) Influence of chip breaker location and angle on chip form in turning low carbon steel. Int J Mach Mater 5(4):452-475

7. Qibiao Y, Zhanqiang L, Bing W (2012) Characterization of chip formation during machining 1045 steel. Int J Adv Manuf Technol 63(9-12):881-886

8. Jawahir IS, Oxley PLB (1988) The tool restricted contact effect as a major influencing factor in chip breaking: an experimental analysis. CIRP Ann Manuf Technol 37(1):121-126

9. Pacella M, Axinte DA, Butler-Smith PW, Shipway P, Daine M, Wort C (2015) An assessment of the wear characteristics of microcutting arrays produced from polycrystalline diamond and cubic boron nitride composites. J Manuf Sci Eng 138(2):210011210016

10. Pacella M, Axinte DA, Butler-Smith PW, Daine M (2014) On the topographical/chemical analysis of polycrystalline diamond pulsed laser ablated surfaces. Proc CIRP 13:387-392

11. Pacella M, Axinte DA, Butler-Smith PW, Fay MW (2014) FIB/ $\mathrm{TEM} /$ EELS micro/nanometric investigations of the effects of laser ablation on the diamond/binder structure in polycrystalline diamond composites. J Mater Process Technol 214(5):1153-1161

12. Pacella M, Axinte DA, Butler-Smith PW, Fay MW (2015) The allotropic transformation of polycrystalline cubic boron nitride structures resulting from the thermal effects of pulsed laser ablation. Diam Relat Mater 59:62-68

13. Pacella M, Nekouie V, Badiee A (2019) Surface engineering of ultra-hard polycrystalline structures using a nanosecond $\mathrm{Yb}$ fibre laser: effect of process parameters on microstructure, hardness and surface finish. J Mater Process Technol 266:311-328

14. Fang XD, Jawahir IS (1991) On predicting chip breakability in machining of steels with carbide tool inserts having complex chip groove geometries. J Mater Process Tech 28(1-2):37-47

15. Fang N, Jawahir IS (2003) Analytical prediction of the chip backflow angle in machining with restricted contact grooved tools. J Manuf Sci Eng 125(2):210-219

16. Fatima A, Mativenga PT (2013) A review of tool-chip contact length models in machining and future direction for improvement. Proc Inst Mech Eng Part B J Eng Manuf 227(3):345-356

17. Poletika MF (1969) Contact loads on tool faces. Moscow Mach (in Russian)

18. Jiang YC, Zhang YZ (1984) Experimental research of the chip flow direction and its application to the chip control. Ann CIRP 33(1): $81-84$

19. Ganesh N, Kumar MU, Kumar CV, Kumar BS (2014) Optimization of cutting parameters in turning of En 8 steel using response surface method and genetic algorithm. Int J Mech Eng 3(2):75-86

20. Dhar NR, Islam MW, Islam S, Mithu MAH (2006) The influence of minimum quantity of lubrication (MQL) on cutting temperature, chip and dimensional accuracy in turning AISI-1040 steel. J Mater Process Technol 171(1):93-99

21. Gürbüz H, Kurt A, Șeker U (2012) Investigation of the effects of different chip breaker forms on the cutting forces using artificial neural networks. Gazi Univ J Sci 25(3):803-814 
22. Kuo RY, Junz Wang JJ, Lee RN (2018) Effect of insert groove geometry on chip breaking performance. J Mech 34(1):67-73

23. Li XB, Zheng JM, Li Y, Kong LF, Shi WC, Guo B (2019) Investigation of chip deformation and breaking with a staggered teeth BTA tool in deep hole drilling. Metals 9(46):1-14

24. Barry J, Byrne G (2002) The mechanisms of chip formation in machining hardened steels. J Manuf Sci Eng 124(3):528-535

25. Kang Z, Fu Y, Ji J, Tian L (2017) Numerical investigation of microtexture cutting tool on hydrodynamic lubrication. J Tribol 139(5):0545021-0545028

26. Dumitru G, Romano V, Weber HP, Sentis M, Marine W (2002) Femtosecond ablation of ultrahard materials. Appl Phys A Mater Sci Process 74(6):729-739
27. Odeshi AG, Bassim MN, Al-Ameeri S, Li Q (2005) Dynamic shear band propagation and failure in AISI 4340 steel. J Mater Process Technol 169(2):150-155

28. Nakayama K (1977) On the formation of saw-toothed chip in metal cutting. Int J Jpn Soc Precis Eng 43(505):117-122

29. Davies MA, Chou Y, Evans CJ (1996) On chip morphology, tool wear and cutting mechanics in finish hard turning. CIRP Ann Manuf Technol 45(1):77-82

Publisher's note Springer Nature remains neutral with regard to jurisdictional claims in published maps and institutional affiliations. 